\title{
Next Generation of Web-based Information Management System for National Volunteer Service
}

\author{
Feng Tian ${ }^{1,2, a^{*}}$, Yundong Yao ${ }^{2,3, b}$, Tian Lan ${ }^{1,2, c}$, Qinghua Zheng ${ }^{2,3, d}$, \\ Jingjing Kou ${ }^{4, e}$, Haifei Fu ${ }^{4, f}$, and Nazaraf Shah ${ }^{5, g}$ \\ ${ }^{1}$ Systems Engineering Institute, Xi'an Jiaotong University, Xi'an, China \\ ${ }^{2}$ Shaanxi Province Key Lab. of Satellite and Terrestrial Network Tech. R\&D, Xi'an, China \\ ${ }^{3}$ Department of Computer Science and Technology, Xi'an Jiaotong University, Xi'an, China \\ ${ }^{4}$ Zhongqinghuayun New media Technology Ltd, Beijing, China \\ ${ }^{5}$ Department of Computer Science and Technology, Coventry University, Coventry, UK \\ a ftian@sei.xjtu.edu.cn, b yaoyundongyyd @stu.xjtu.edu.cn, ' lantian92@stu.xjtu.edu.cn, \\ d qhzheng@mail.xjtu.edu.cn, ${ }^{\mathrm{e}}$ kjj@cyol.com, ${ }^{\mathrm{f}}$ fuhf@cyyun.com, ${ }^{\mathrm{g}}$ aa0699@coventry.ac.uk
}

Keywords: volunteer service; volunteer information management; next generation

\begin{abstract}
This paper presents a survey about the existing web-based information management system for volunteer services. Based on the survey results the paper highlights the new features and functions of the next generation of the volunteer service system. A prototype system incorporating these features and functions has also been implemented and presented.
\end{abstract}

\section{Introduction}

Voluntary service is an important indicator of the development of civilization in modern society [1], and makes an important part of society service which is connected with government service and market service. However, after Chinese voluntary service information systems have experienced the initial disorderly development period, it is necessary to accomplish the specialization of the information platform for voluntary service and reform the voluntary service process. It is important to take full advantage of technologies such as the Internet, intelligent computing, cloud computing and so on to make the voluntary service intelligent, personalized and seamlessly connected. These requirements involve the following four basic scientific issues:

1) How to construct the basic software and hardware platform meeting the needs of large scale data storage and interaction for voluntary service as well as voluntary production and consumption.

2) How to manage and coordinate various voluntary organizations (such as unregistered civil voluntary organization, Non-Governmental Organizations, legal organizations registered in the Civil Affairs Bureau etc.) uniformly by combining the Internet, information technology and intelligent technology to make the voluntary service information shareable and interoperable, and to establish a national volunteer credit system and be able to effectively deal with the large scale sudden disasters.

3) How to accomplish the personalized recommendation for voluntary activities, which takes an overall consideration of the factors such as the volunteers' interest and the time interval they are available in, to better meet the needs of volunteers participating in voluntary activities recommended to them ( two-way recommendation problem).

4) How to accomplish the transparent track of voluntary service release, recruitment implementation and the comprehensive evaluation of voluntary service like social value evaluation of public voluntary service.

This paper first introduces the state of art of the web-based information management system for voluntary service at home and abroad. Then, we describe several key technologies required by the 
next generation of the web-based information management system for voluntary service mainly from the technical level. Finally, a prototype system is presented.

\section{Related Work}

Currently, some well-known voluntary organizations at home and abroad, such as United Nations voluntary organization, EU voluntary organization, worldwide helpers etc., all achieve a mature and stable development. With the rapid development of the computer technology and Internet, each voluntary organization establishes its corresponding volunteer website to facilitate the management of volunteers and volunteering information. We have reviewed 16 well-known domestic and foreign volunteer organizations (including 11 foreign websites and 5 domestic websites) and their corresponding website addresses (http://www.volunteeringinamerica.gov/, http://www.unv.org/, http://www.europeanvoluntaryservice.com/, etc.).

According to the statistics on the functions provided by these volunteer websites, we sort the functions by the frequency and ratio of their occurrences. Then we divide these website functions into basic functions (the probability of their occurrences is greater than $50 \%$ ), common functions (the probability of their occurrences is between $20 \%$ and $50 \%$ ) and low-frequency functions (the probability of their occurrences is less than 20\%). Among them, basic functions are the fundamental functions of a website system, ensuring the normal development and promotion of voluntary activities, and managing volunteer information, such as navigation bar, link with social networks, news, search and so on. Common functions are provided for website users by volunteer websites, related to voluntary service information and frequently used, such as log in, FAQs, learning, training and so on. Low-frequency functions are functions with a small frequency of occurrences in the existing voluntary service information websites, which are ignored due to either legal reasons or cultural differences, such as find appropriate activities, find voluntary partners, and legal help etc. In addition, platforms and systems combined with new applications like mobile client have started to provide services in websites of some voluntary organizations. According to the present situation of the voluntary service information management system based on Web, there exist the following problems: low ability to deal with large scale data and interaction; poor interconnection/interoperability between websites; lack of personalized recommendations; difficulty in project transparency tracking.

Nowadays, the implementation of the volunteer service management platform is mostly based on PHP, and J2EE etc. [1-6]. For example, MacDonald [1] designed a volunteer website specific to adults ensuring ease and minimal confusion as much as possible. Lan $\mathrm{Yu}$ [6] proposed a volunteer management platform based on the SOA architecture.

To sum up, considering the factors such as large population in China, vast territory and so on, and the whole society is in the initial stage of the formation of voluntary culture. The voluntary service information management system should have the following functions: support of large scale data interaction, interconnection/interoperability, intelligent matching for voluntary activities, and evaluation and tracking of voluntary service activities. However, there are few research efforts on the four issues proposed in the introduction of this paper.

\section{The Proposed Architecture and Key Technologies of the Next Generation of the Web-based Information Management System for National Volunteer Service}

The architecture of the next generation of the information management system for national volunteer service is presented in Fig. 1. It takes advantage of resource management and storage architecture of cloud computing to meet the requirements of large scale data storage for the production and consumption of voluntary activities and volunteers. It also utilizes the elastic extension and environmental perception of cloud computing to meet the requirements of data exchange and massive interaction between different mobile and heterogeneous networks, especially the requirements of large scale information exchange and service requests in response to the occurrence of large scale sudden disasters. Next, with this platform, employs data mining, 
information extraction, video and audio understanding and other technologies for real-name authentication, data sharing, interaction, analysis, evaluation of individual behavior, transparent track of voluntary donation and social value evaluation of public voluntary service. Furthermore, with the support of these key technologies, four subsystems named volunteer community, national volunteer service support system, socialized transparent donation and volunteers' return can be integrated into a unified portal.

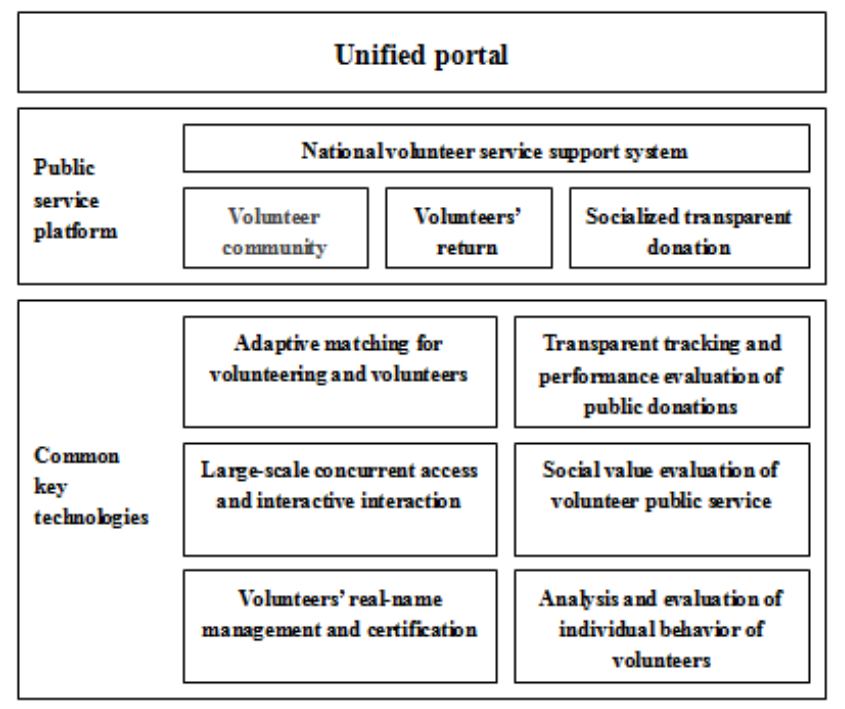

cloud computing platform for national vohnteer public service

Fig. 1. The architecture of the next generation of the web-based information management system for national volunteer service.

Compared with current systems, the features of the next generation of the information management system for national volunteer service are listed as follows:

(1) Storage and management of large scale resources of volunteer service. With the participation of all social strata, and the continuous development of various volunteer activities, the volume of the resources that volunteer service produces and consumes continue to grow. Therefore, the architecture of the next generation of the information management system for national volunteer service must have strong scalability of resource storage and effective interaction service ability in order to solve the problem of massive resource storage and management.

(2) Unified real name authentication and standardization of volunteer service information. Only using a unique identification code of volunteers and standardizing the volunteer service information can ensure the exchange and interaction of all kinds of information of volunteer service and volunteers between various volunteer organizations and their systems, and guarantee the authenticity and accuracy of the information. This will lay a solid foundation for a national volunteer management and credit system.

(3) Intelligent discovery of volunteers and volunteer activities. Existing technologies relying on manually browse and keyword match to discover volunteer activities or volunteers are inefficient and are difficult to achieve the goal of fine matching between volunteers and voluntary activities. First, adopt the technology of personalized user behavior analysis to analyze the characteristics of volunteers. In other words, based on data mining and large data analysis technology, we can find common features of thousands of volunteers and individual differences between them. By formatted representation and semantic analysis of volunteer activities, we can obtain accurate perception of the interests of volunteers/donors and the needs of recipients. Finally, bidirectional adaptive recommendation between volunteer and volunteer activities can be realized.

(4) Transparent track of volunteer service. For creating the "sunshine-like-transparent platform" of volunteer service, it must be ensured that the contents of volunteer service (donating service and 
goods) are open publicly and can be supervised by the public, the process can be tracked and the results of service can be measured.

(5) Comprehensive/integrated social value evaluation of volunteer service. By analyzing the collected data, we can quantify the quantity and quality of the volunteer service, in order to discover significant problems of the public service system in time and quantitatively reflects the contribution of the spirit of selfless dedication advocated by the volunteer service to the whole social value.

\section{Prototype System}

We use 2 DELL R910 as computing nodes and each node is configured with four E7-4870 Intel and 256GB memory. We also use 4 DELL R720 as storage nodes and each node is configured with six $2 \mathrm{~T}$ hard drivers. We use 2 Cisco 2960s switches. One is used for storage network and after dividing VLAN, the other is used for data exchange between intranet and extranet.

Each DELL R910 is configured with CentOS 6.5 and KVM to implement virtualized infrastructure. Each DELL R720 is configured with CentOS 6.5 and Ceph to implement the distributed file system used for storing virtual machine images. Meanwhile, according to the requirement, we customize and optimize the Folsom version of OpenStack and configure Nova compute, Noca Network and the other components for the operation, maintenance, management and scheduling of the cloud platform. OpenStack, KVM and Ceph are the core components of our cloud platform.

Based on the virtual machines provided by our cloud computing platform, we set up the development and testing environment, including configuring the application server cluster composed of 3 Tomcat middleware virtual machines to provide Java containers for business logic, configuring the database cluster composed of 1 Amoeba virtual machine and $2 \mathrm{Mysql}$ database virtual machines to implement the load balance of databases and make it possible to scale out the databases, based on Amoeba's ability of Horizontal Partitioning of data. We also configure the web server cluster composed of 3 Mginx virtual machines to provide responses to the static page requests and in the front end configure a virtual machine with HaProxy which can shunt the users' requests in order to implement the load balance of application layer. Meanwhile, we configure a virtual machine which runs NTP service to synchronize time of each server (virtual machines and physical servers). In the developing and testing environment, the future need of the scalability is fully considered and the three aspects of the MVC model are implemented respectively. It is the complete simulation of the production environment.

In terms of the standardization of unified real name authentication and volunteer service information, the most difficult problem is sharing and interaction/interoperability of volunteer service information. Only by overall design and overall arrangement, the data interface standards of heterogeneous systems can be accomplished. According to the investigation and research of our research group, we are now going to apply our achievement for national standards and industry standards. The standards of data sharing and interaction in the volunteer information management system in China's Ministry of Civil Affairs have been in the second stage of consultation. A screen shot of real name authentication is shown in Fig. 2.

In the technology of intelligent discovery of large scale volunteers and voluntary activities, our group analyzes the real data of existing volunteers and voluntary activities and finds out that the features which are "geographically closely participating" and "participating together". Based on these features, we propose an estimation algorithm about the rating matrix-based collaborative filtering algorithm and achieve the bidirectional recommendation of the volunteers and the voluntary activities in the framework of large scale collaborative filtering algorithm.

In terms of the comprehensive social value evaluation of voluntary service, we propose a system for volunteer service comprehensive value evaluation from view of economic value and non-economic value (social civilization, and resource utilization and collaboration). Besides, we determine the weight of indexes through hierarchy analysis method, which can reflect the macroscopic state of national volunteer service combined with the visualization technology. 


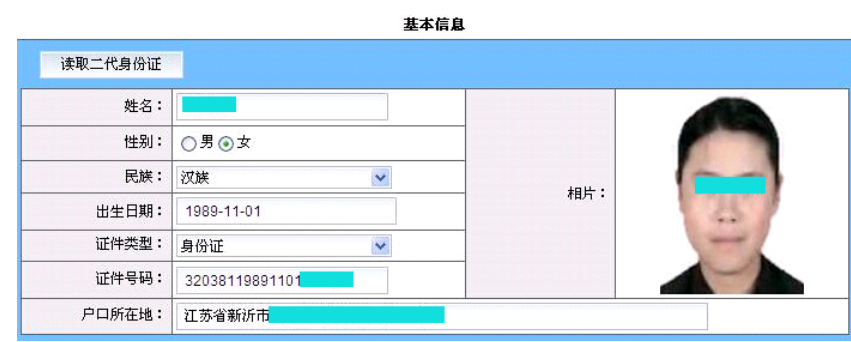

Fig. 2. Users' basic information collected by a portable ID card reading equipment.

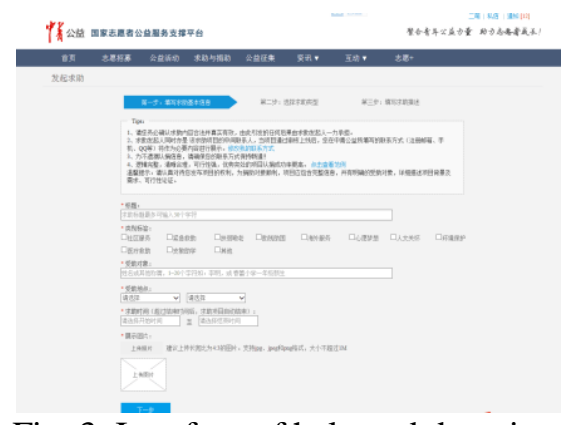

Fig. 3. Interface of help and donation.

To maintain transparent track of voluntary activities, by extracting the needs of help seekers and discovering the interest of service providers, the intelligent matching between them can be implemented. And then, tracking the whole process of voluntary service can make every working step queried and tracked. it also lays emphasis on the feedback and evaluation of the follow-up voluntary activities. A screenshot of voluntary donation steps is shown in Fig. 3.

\section{Summary}

This paper presents a survey about the existing web-based information management system for volunteer service and points out essential new features and functions of the next generation of the system. A prototype system these features is introduced.

Future work will focus on how to promote the application of the proposed system, and to promote the implementation of the proposed standards in all kinds of volunteer information management systems, in order to achieve the information sharing of national volunteers and voluntary activities. The important aspects, such as algorithm for large scale disasters oriented voluntary activity recommendation and resource scheduling will be researched.

\section{Acknowledgement}

This research was partially supported by the National Key Technologies R\&D Program of China under Grant No.2013BAK09B01, the National Natural Science Foundation of China under Grant Nos. 61428206 and 61472317, the Ministry of Education Innovation Research Team No. IRT13035.

\section{References}

[1] McDonald M L, "Volunteer Website for the Older Adult, Proceeding of International Conference on Universal Access in Human-computer Interaction: User \& Context Diversity-volume," Lecture Notes in Computer Science, Volume 8010, 2013, pp 151-155.

[2] Fernandez L S, "Volunteer management system design and analysis for disaster response and recovery," System Science, 2007.

[3] Chalasani S, Baldwin D, Sounderpandian J, et al, "Information System for a Volunteer Center: System Design for Not-For-Profit Organizations, Cases on Information Technology Planning," Design and Implementation, M. Khosrow-Pour Eds.IGI Global, USA, 2006.

[4] Moy L, "Tapping Global Resources: A Guide to Involving and Managing Online Volunteers," Journal of Volunteer Administration, 2002, 20(2):47-52.

[5] Shunqian Lin, "C/S framework-based youth volunteer management system," University of Electronic Science and Technology of China, Master thesis, 2012.

[6] Lan Yu, "Development of SOA-based Yi'de volunteer service management system," Fudan University, Master thesis, 2011. 